\title{
Temporally Resolved HyperSpectral Analysis vs Radiation Damage in Hard and Soft Matter
}

\author{
Nestor J. Zaluzec ${ }^{1}$ \\ ${ }^{1}$ Photon Sciences Division, Argonne National Laboratory, Il, USA
}

The effects of high energy electrons introducing radiation damage to the microstructure of both hard and soft matter have been studied for many decades. These effects cover a large range of processes including: structural damage via radiolysis and atomic displacement [1-3]; charging and heating [4-5]; as well as mass loss by sputtering, or gain by contamination [6-7]. Similarly the effects on elemental redistribution in the Analytical Electron Microscope (AEM) have been long recognized [8-9], however, the advances in detector technology [10] and data acquisition schemes (DAQ) such as time resolved hyperspectral imaging (HSI) and cryo-enabled specimen holders [11] have allowed us to being to study systems which in the past were untenable, however, the effects have yet to be eliminated.

The complicated nature of these combined effects listed above are illustrated in Figures 1-3. In this work the specimen investigated was a commercially produced XRF multi-element standard C10 used by the synchrotron community and was purchased from AXO Dresden GmbH. It consists of 6 different deposited reference elements $\left(\mathrm{Pb}\left[84.9 \mathrm{ng} / \mathrm{mm}^{2}\right.\right.$ ], $\mathrm{La}$ [121.4], $\mathrm{Pd}$ [23.3], Mo [8.6], $\mathrm{Cu}$ [22.2], $\mathrm{Fe}[43.9]$, \#RF17-200-S4216-19) upon a $200 \mathrm{~nm}$ thick SiNx transmission window on a Si support. The specimen was studied in a ThermoFischer Talos AEM at $200 \mathrm{kV}$ using a SuperX SDD detector system at a beam current of $\sim 120 \mathrm{pA}$ using a nominal $5 \mathrm{~nm}$ probe size. Multi-frame drift corrected temporally resolved hyperspectral data sets $(800 \times 800$ pixels $)$ were recorded at $40 \mathrm{usec} /$ pixel for 1000 frames. For application to XRF studies this "standard" is advertised as homogenous at $~ 30$ micron scale to the $1 \%$ level. Relative to high resolution AEM studies the specimen is extremely inhomogenous as can be seen in Figure 1a which presents a HAADF image taken under low dose conditions at room temperature. The elemental inhomogeneity is confirmed in Figure 1b, which is an HSI background corrected image of the $\mathrm{Cu}$, La, and $\mathrm{Pb}$ distributions, while $1 \mathrm{c}$, shows the sum spectra over the entire field of view (FoV). Time resolved windows of the distribution change with time are shown after $4 \mathrm{msec}$ and $40 \mathrm{msec} / \mathrm{pixel}$ in Figures 2 and 3. Interestingly the sum spectra over the FoV changes very little over time, except for the Carbon which increases slowly due to contamination build up. As the specimen is delivered in a gelatin capsule this is not surprising, and would be not-detectable by most synchrotron XRF measurements. The contamination appears to act as a containment layer and although at the local nanoscale the various atomic species redistribute, they do not sputter out of the FoV. This is markedly different from specimens which do not contaminate where elemental sputtering has been well documented $[8,9,11]$. While mass redistribution in the AEM, for beam sensitive materials can be both predicted [7] and observed, the increase in solid angle and the ability to perform time resolved HIS, allows today's temporally resolved experiments to investigate some systems which in the past were simply untenable [12].

References:

[1] M Isaacson in "Principles and Techniques of Electron Microscopy V 7", p. 1.

[2] KH Downing and RM Glaeser, Ultramicroscopy 20 (1996), p. 269.

[3] DL Medlin and DG Howitt in "Microbeam Analysis" (SanFrancisco Press) p. 272.

[4] J Cazaux, Utlramicroscopy 60 (1995), p. 441.

[5] RF Egerton et al., Micron 35 (2005), p. 399.

[6] J Hren in "Introduction to Analytical Electron Microscopy" (Plenum Press) p. 481. 
[7] CR Bradley and NJ Zaluzec, Ultramicroscopy 28 (1989), p. 335.

[8] JM Mansfield et al., Ultramicroscopy 21 (1987), p. 13.

[9] WH Massover et al., Micro. Microanal. 18(S2) (2012), p. 1212.

[10] NJ Zaluzec, Microsc. Microanal. 20 (2014), p. 1318.

[11] M Ovsyanko et al., Microsc. Microanal. 24(1) (2018), p. 776.

[12] This research was funded in part by Laboratory Directed Research and Development (LDRD) funding No. 2017-153-N0 at Argonne National Laboratory by the U.S. Department of Energy, Office of Science, Office of Basic Energy Sciences, it employed resources in the Center for Nanoscale Materials, an Office of Science user facility.

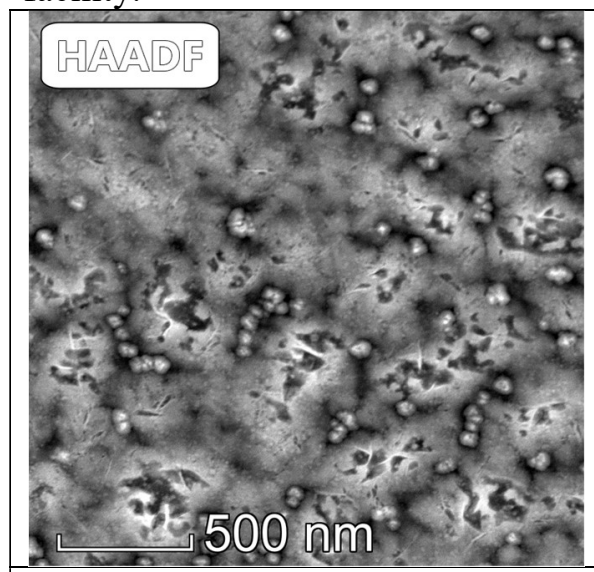

Figure 1. a) HAADF @ $0.4 \mathrm{msec} /$ pixel

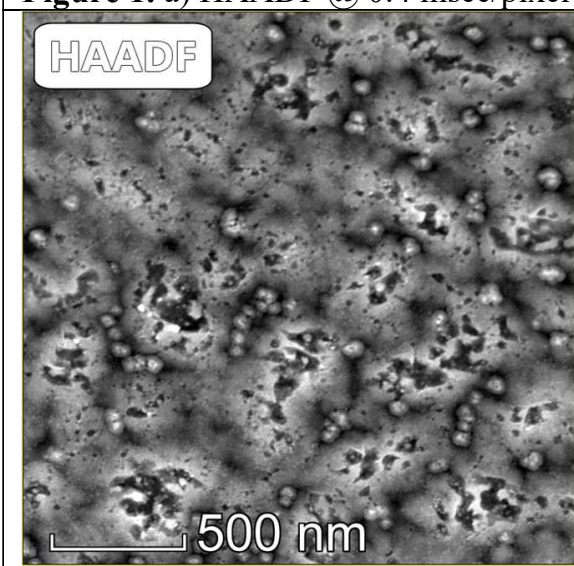

Figure 2. a) HAADF@ $4 \mathrm{msec} / \mathrm{pixel}$

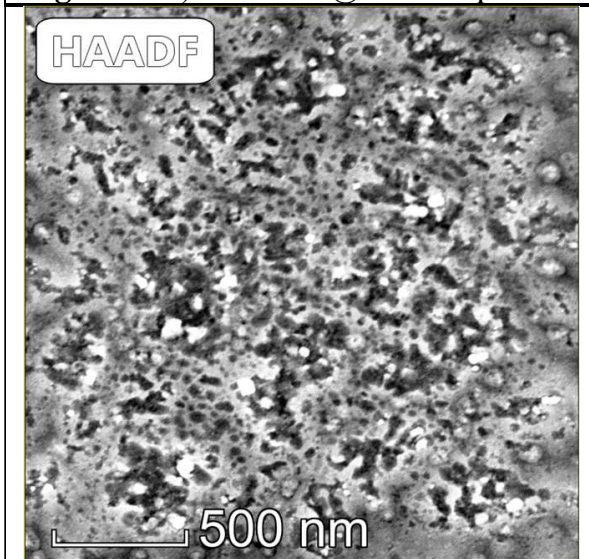

Figure 3. a) HAADF @ 40 msec/pixel

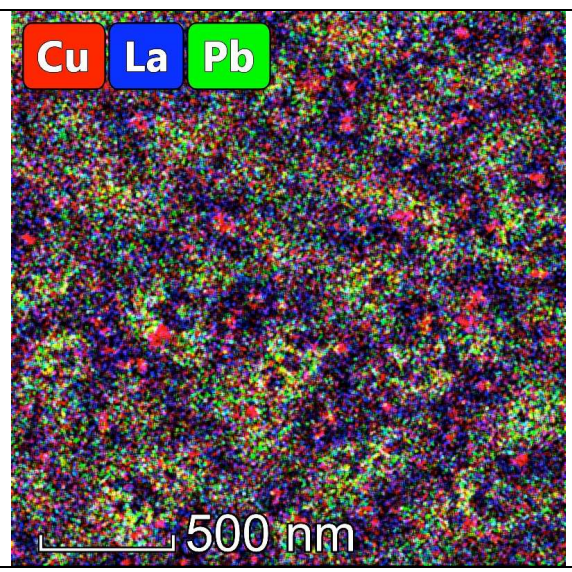

Fig 1.b) $\mathrm{CuLaPb}$ HSI @ 0.4 msec/pixel

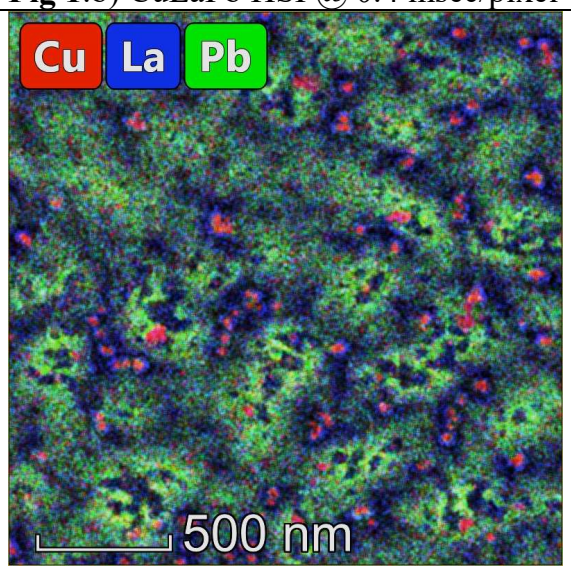

Fig 2.b) CuLaPb HSI @ 4 msec/pixel

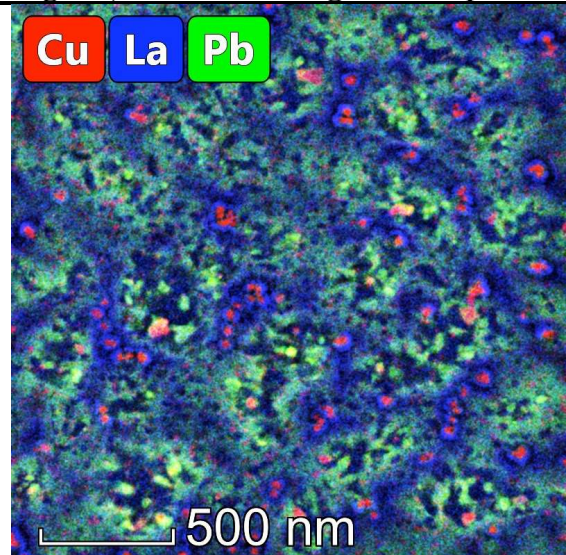

Fig 3. b) CuLaPb HSI @ 40msec/pixel

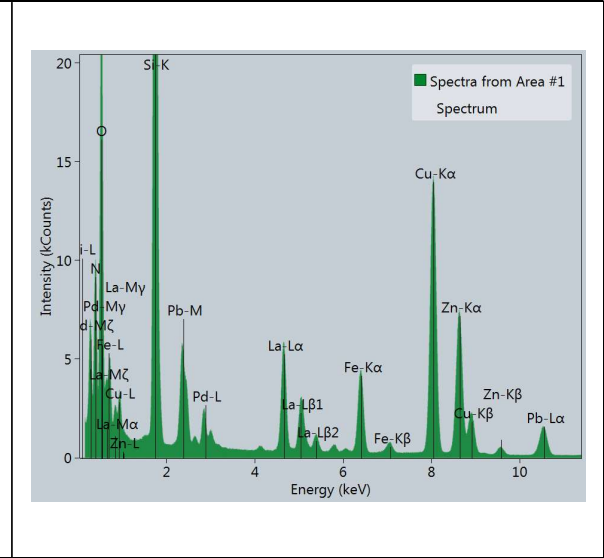

Fig 1. c) SumSpectra @ 0.4 msec/pixel

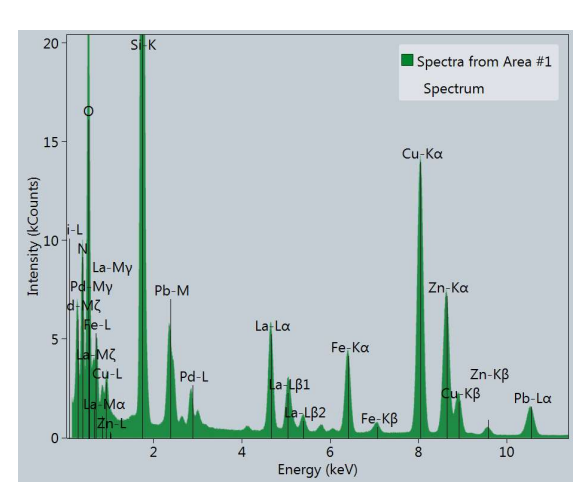

Fig 2. c) SumSpectra@4 msec/pixel

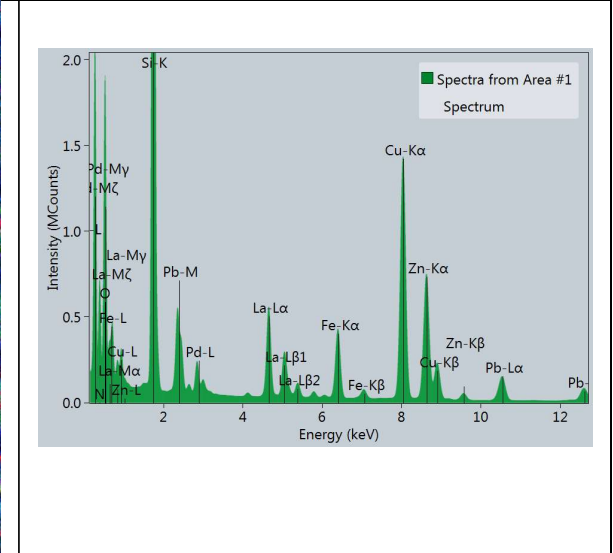

Fig 3. c) SumSpectra @ 40 msec/pixel 